\title{
A Collaborative Approach to Enhance CBIR Performance using DCT, DST and Kekre's Transform
}

\author{
Avanish Tiwari \\ RITS, Bhopal, M.P. India
}

\author{
Anurag Jain \\ RITS, Bhopal, M.P. India
}

\begin{abstract}
Content based Image retrieval is a process which will enhance the image searching accuracy. Many researchers are working on image processing. CBIR approach uses different techniques such as colour, texture and shape, by processing this feature vector is generated and comparison is done. CBIR approach can be used in many places such as search engines, patent registration, face detection etc. CBIR approach can also be used for security. It can encrypt and decrypt the image content to provide security. Stenography along with CBIR can generate algorithm which makes data more secure. CBIR can help in many fields only by referring image content. In this paper new approach "Hybrid approach" is implemented. Hybrid approach is a combination of different transforms. Combination of DCT, DST and kekre's transforms are used for feature vector generation. For image matching and distance calculation, two methods are used in this paper known as Euclidian distance and absolute distance method. In this paper, different transforms are combined to generate a hybrid approach. Results of different hybrid approaches are compared in this paper. It includes comparison of all the algorithms based on their performance by comparing different performance parameters such as precision and recall to determine which algorithm is providing the best result.
\end{abstract}

\section{General Terms:}

CBIR, Pattern Matching, Image Retrieval, Hybrid transform, Collaborative transform

\section{Keywords:}

CBIR, Feature Vector, Transform, Sectorization, Spatial Domain, Frequency Domain, Similarity Measures, Hybrid transform, Collaborative transform, combining different transforms

\section{INTRODUCTION}

Whole world is craving for the best security method. Trademark experts and patent registrars are searching for the best way to find out the efficient method for searching and comparison of image. Not only had this but today computer professionals also stuck how to manage day by day rapidly growing digital data. Searching image is a major problem in today's image era where only social networking apps are generating millions of image in a day. Searching an image was tedious job from ancient time. Keyword based searching lags where a similar image comes and you have to find out image with very minor difference. [1][2] Keyword searching approach is not able to distinguish between images and categorize them based on image feature like colour, shape and texture. Content based image retrieval technology is used to manage digital image based on their content. [3] The technology which is used to categorize digital image based on visual content is CBIR. Because of this characteristic, CBIR becomes famous in many scientific communities. One problem with all current approaches is the reliance on visual similarity for judging semantic similarity, which may be problematic due to the semantic between low-level content and higher-level concepts. While this intrinsic difficulty in solving the core problem cannot be denied, it is believed that the current state-of-the-art in CBIR holds enough promise and maturity to be useful for real-world applications if aggressive attempts are made. Before keyword searching, some spatial domain searching was also used to generate image matching result. Histogram [17][14] matching was implemented which was using image histogram enhancement technique to match the resultant output. But that also have limitation such as when different class image have same colour then the image histogram will be same, which will generate improper result. So frequency domain [20] can play better role to generate the feature vector.

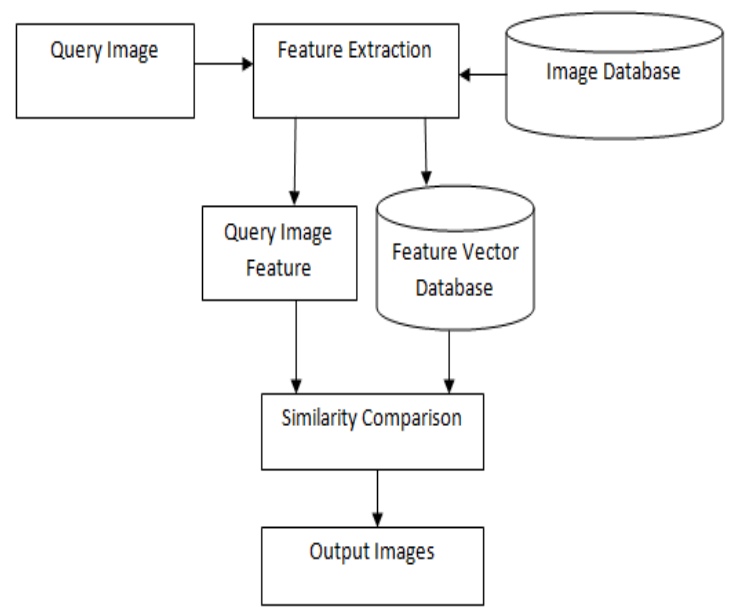

Fig 1: CBIR processing model

Fig 1 shows the basic architecture of content based image retrieval system. Image database will be given to feature extraction where feature extraction algorithm will get applied and resultant value will be stored in feature vector database.[5][13] Whenever CBIR system will get query image from the user, it will be given to feature extraction where feature extraction algorithm will be applied and query feature vector will be prepared. This query image feature vector and feature vector database will be used to compare similarity and most relevant image will be displayed as an output.

\section{DISCRETE SINE TRANSFORM}

Discrete sine transform is implemented with an innovative idea. In this, DST transform was sectorized [8] into 4,8,12 and 16 sectors of images to generate the feature vector in the content based image retrieval system. [1][2] The work is experimented over even-odd row/column component planes of DST transformed image. Performance of this method was measured on the basis of precision and recall values. The performance of DST sectorization with augmentation for both planes gives good result of retrieval, on an average $45 \%$ when using the Euclidian distance as similarity measure and $46 \%$ when using the sum of absolute difference as similarity measure. Thus it is advisable to use the sum of absolute difference as similarity measure because of its simplicity and 
less computational complexity as compared to Euclidian distance. [6][7]

\section{DISCRETE COSINE TRANSFORM}

Discrete cosine [20] is used for energy compaction. For same image quality, DCT can give high compression rate as compared to FFT, because cosine basis functions can afford for high energy compaction.

This transformation can be implemented by applying transformation on column and row. Just as the Fourier transform uses sine and cosines waves to represent a signal, the DCT uses only cosine waves. Hence DCT is purely real. The cosine functions are taken over half the interval and this half interval is divided into $\mathrm{N}$ equal parts and each function is sampled at the centre of each part. The discrete cosine transform matrix is formed by arranging these sequences rowwise.

\section{KEKRE'S TRANSFORM}

Kekre's transform will give real and orthogonal transform. This transform generates matrix. To generate this matrix following formula is used

$$
\begin{array}{cc}
1, & x \leq y \\
K(x, y)=-N+(x-1), & x=y+1---(E q 1) \\
0, & x>y+1
\end{array}
$$

This approach will use Eq1 to calculate image transform. For image [f] the transform $[\mathrm{F}]$ is calculated using Eq2.

$$
[F]=[K][f][K]^{t}------(E q 2)
$$

\section{FEATURE VECTOR GENERATION}

Proposed algorithm will use hybrid method of transform to generate feature vector.[10][11] DST and DCT transform will be applied to generate feature vector. It will be classified into sectors to generate feature vector and it will be used for searching and retrieval of an image from database. Same way DST and Kekre's transform, DCT and Kekre's will be applied and result will be classified in sectors to generate feature vector and that feature vector will be used for searching and retrieval of image from database.[5] Transform result will be classified in 4 sector, 8 sector, 12 sector and 16 sectors. Each plane will generate value which will be stored in vector after taking mean value of vector of each sector. Feature vector will be generated by applying absolute distance and Euclidean distance method of similarity measure of the mean value of all sectors. DST-DCT sector 16 for each colour planes i.e. R, G and B are generated. So feature vector will be of size 204 . Same way DST-Kekre's and DCT-Kekre's transform will generate 204 components feature vector.

\section{A. 4 sector}

Table 1: Quadrant assignment rules

\begin{tabular}{|l|l|l|}
\hline Quadrant & $\begin{array}{l}\text { Sign of even } \\
\text { row/column }\end{array}$ & $\begin{array}{l}\text { Sign of Odd } \\
\text { row/column }\end{array}$ \\
\hline I $\left(0^{\circ}-90^{\circ}\right)$ & + & + \\
\hline II $\left(90^{\circ}-180^{\circ}\right)$ & + & - \\
\hline III $\left(180^{\circ}-270^{\circ}\right)$ & - & - \\
\hline IV $\left(270^{\circ}-360^{\circ}\right)$ & - & + \\
\hline \multicolumn{2}{|c|}{ B. 16 sector } \\
\\
$\begin{array}{l}\text { 16 sectors will be generated by dividing } \\
\text { each sector } 4 \text { sectors into four parts. }\end{array}$
\end{tabular}

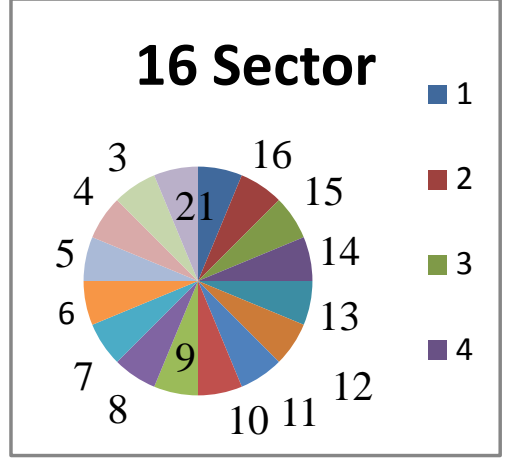

Fig 2: 16 sector division

\section{HYBRID APPROACH}

Since DCT transform is applied to generate result which is better for feature vector generation. It provides $40 \%$ overall matching result. Researchers already tried DST and Kekre's transform to generate feature vector for image comparison. Their enthusiastic work gave sufficient result. But till now not a single researcher tried the combination of different transforms to generate feature vector. In this paper combination of different transform are tried to generate effective feature vector. In this, first a fall discrete sine transform is applied on the image and it will generate a feature vector. Again on the same image content, discrete cosine transform is applied to generate the feature vector. Both feature vectors will be combined and a new feature vector will be saved. In the same way whole database will be generated. When user will fire a query image that will be matched based on the different distance calculation methods to check the exact matching result. Based on this, best matching result will be displayed.

\section{RESULT AND DISCUSSION}

The sample Images of the database of 500 images of 6 different classes such as Human, Tribal, Dinosaur, Bus, Scenery, Beach is shown in the Figure.

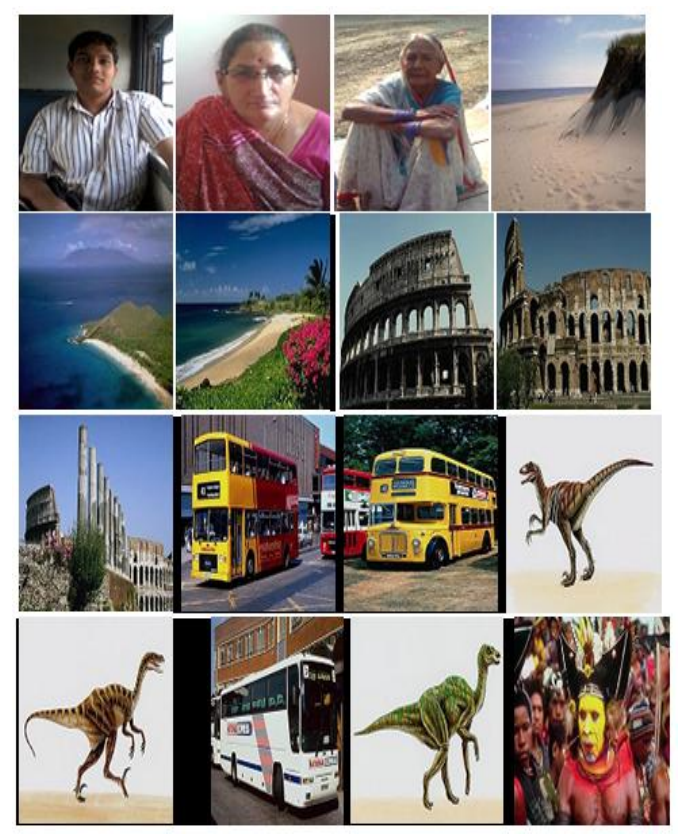

Fig 3: Different class image sample 
Feature database includes feature vectors of all images in the database. Five random query images of each class were used to search the database. The image with exact match gives minimum sum of absolute difference and Euclidian distance. To check the effectiveness of the work and its performance with respect to retrieval of the images overall average Precision

$$
\begin{aligned}
& =\frac{\text { Number of relevant images retreived }}{\text { Total number of images retreived }} \\
& -----E q 3
\end{aligned}
$$

\subsection{DST-DCT Transform}

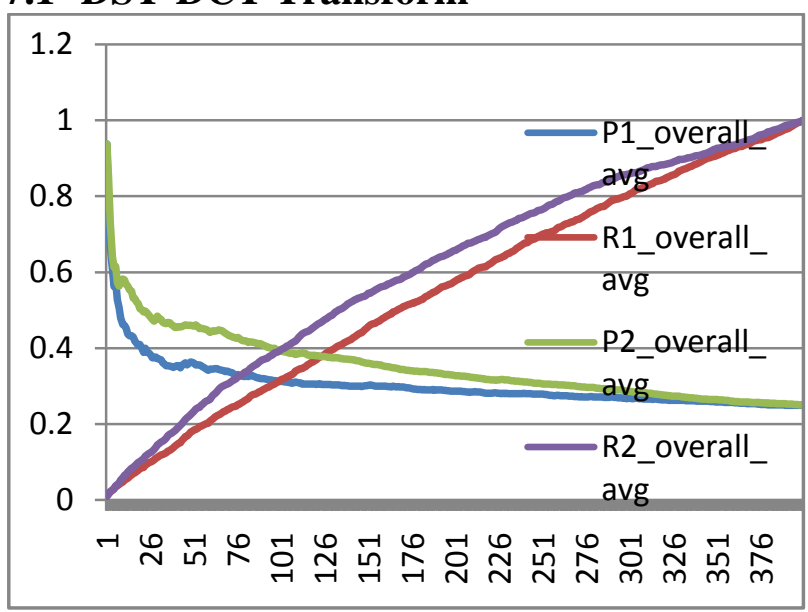

Fig 4: Overall result of DCT-DST transform

\subsection{DCT-Kekre Transform}

\subsubsection{Absolute Distance method}

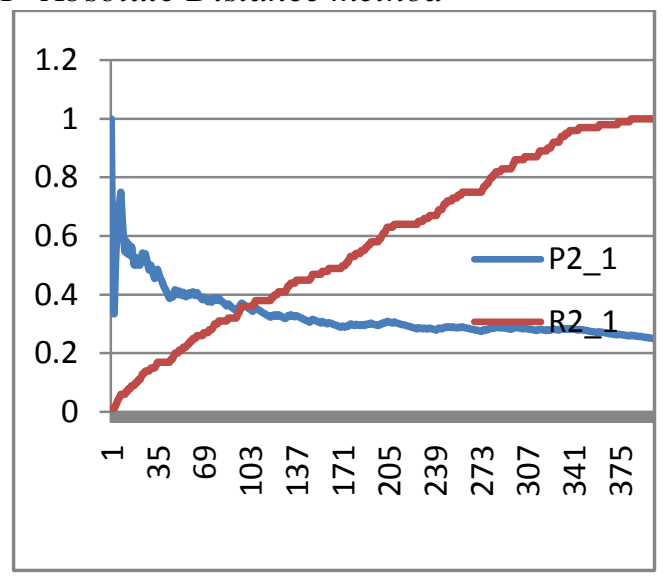

Fig 5: PRCS of class1

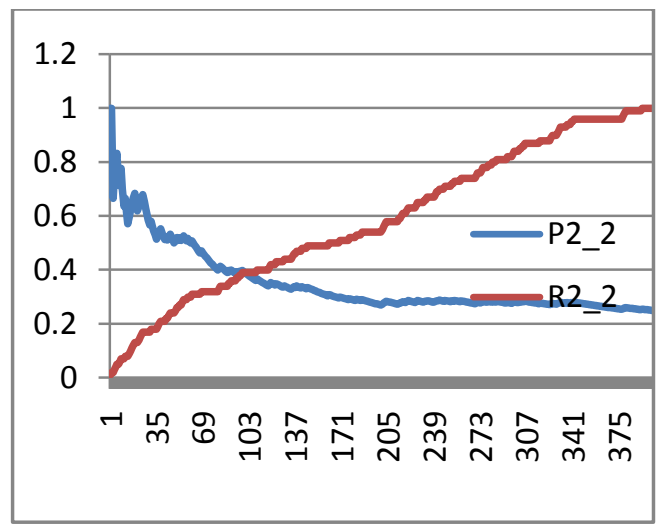

Fig 6: PRCS of class2 precision (Eq 3) and recall (Eq 4) and its cross over values has calculated and plotted class wise. The Equations (1) and (2) are used for precision and recall calculation whilst two new parameters i.e. LIRS (Length of initial relevant string of images) and LSRR (Length of string to recover all relevant images) are used.

$$
\begin{aligned}
& \text { Recall } \\
& =\frac{\text { Number of relevant images retreived }}{\text { Total Number of relevant images in the database }} \\
& -----E q 4
\end{aligned}
$$

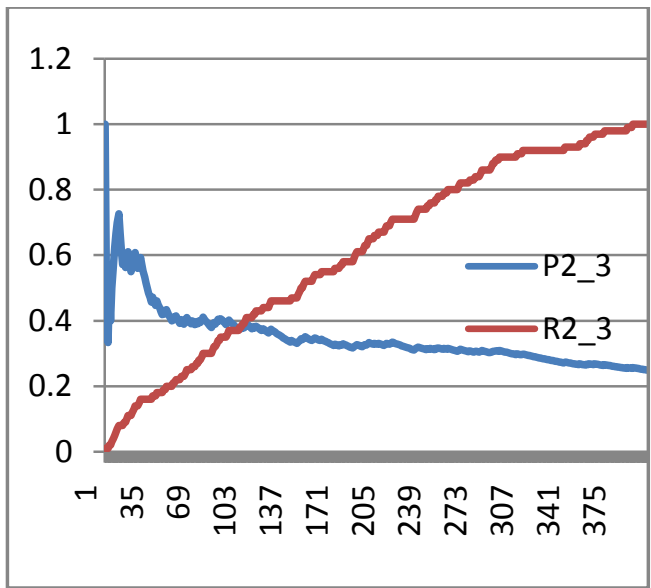

Fig 7: PRCS of class3

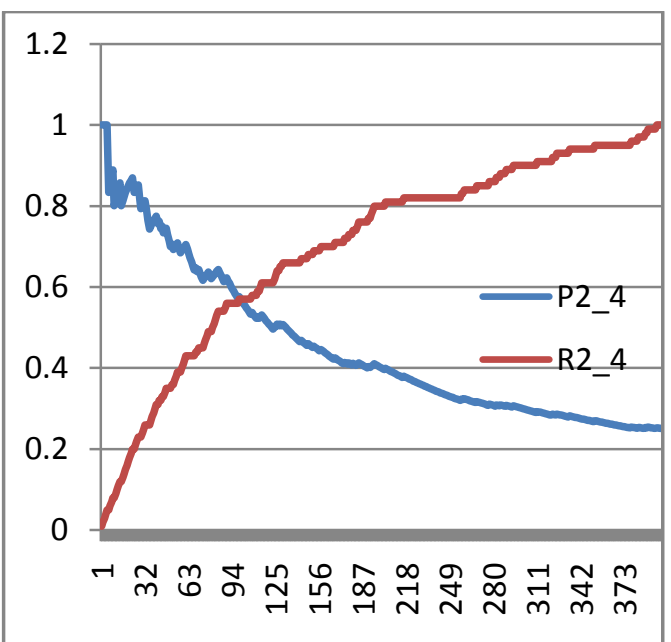

Fig 8: PRCS of class4

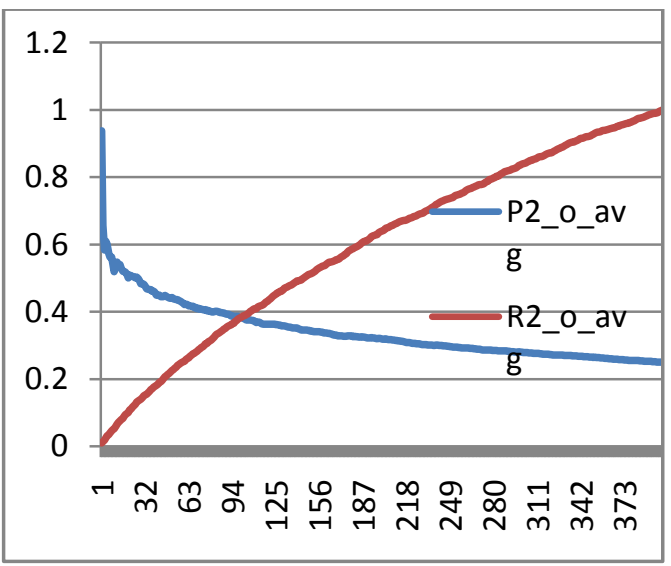

Fig 9: PRCS of overall result 


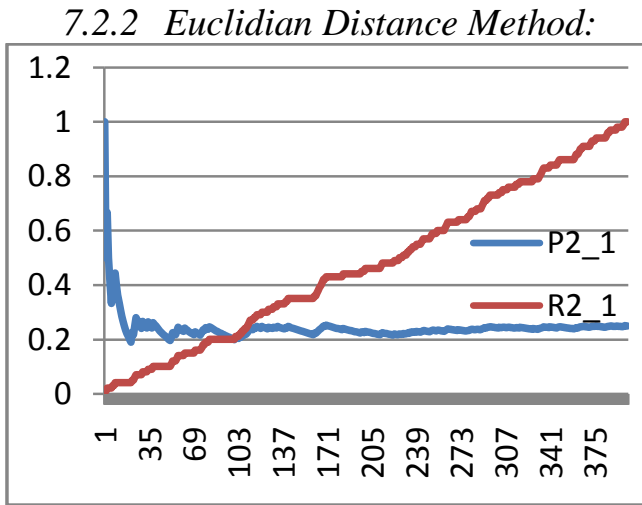

Fig 10: PRCS of class1

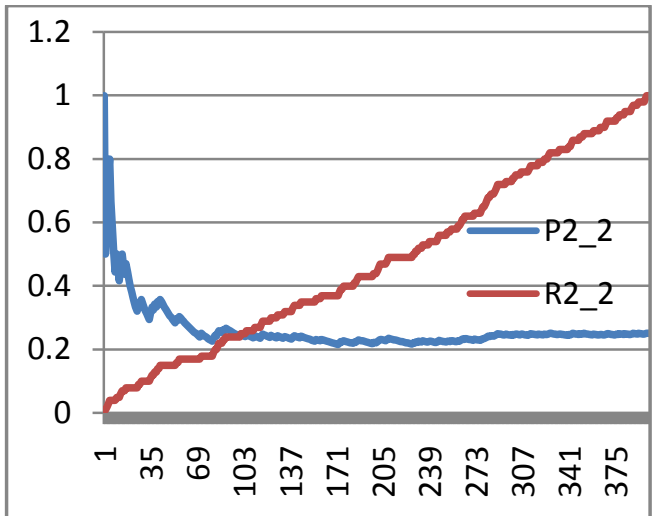

Fig 11: PRCS of class2

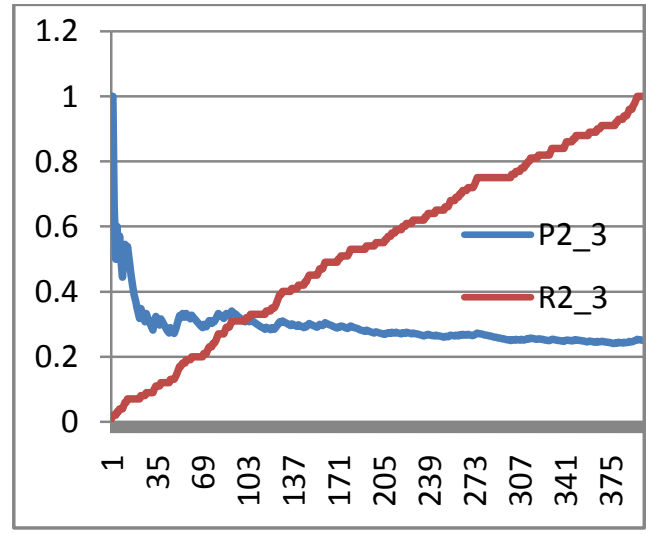

Fig 12: PRCS of class3

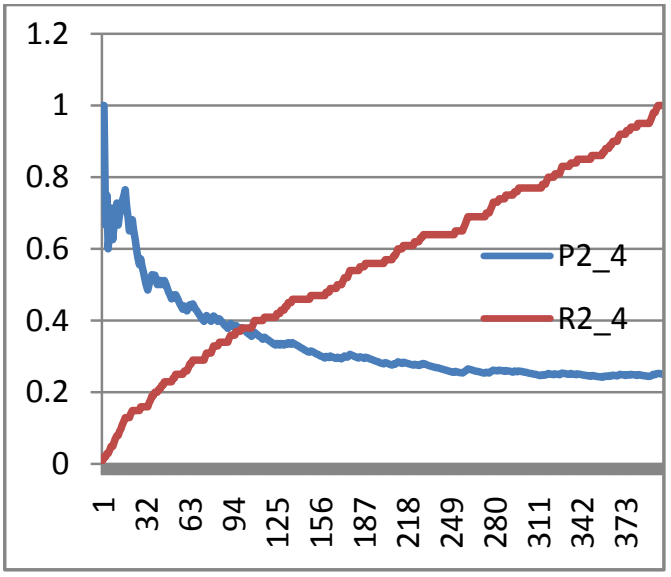

Fig 13: PRCS of class4

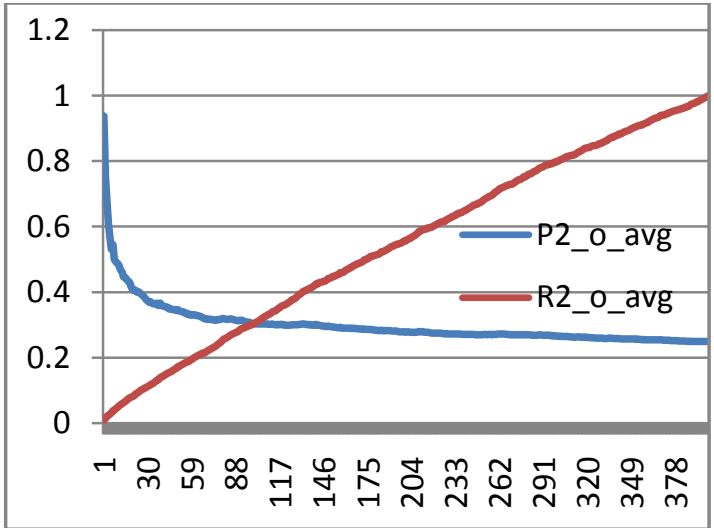

\subsection{DST-Kekre Transform}

7.3.1 Absolute Distance Method

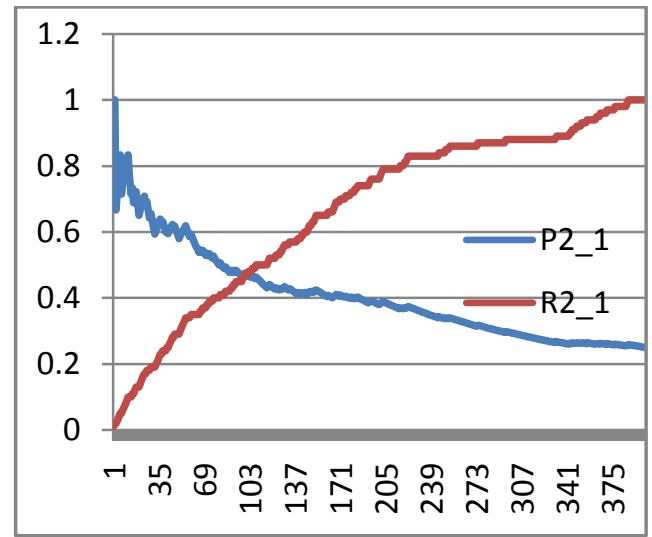

Fig 15: PRCS of class1

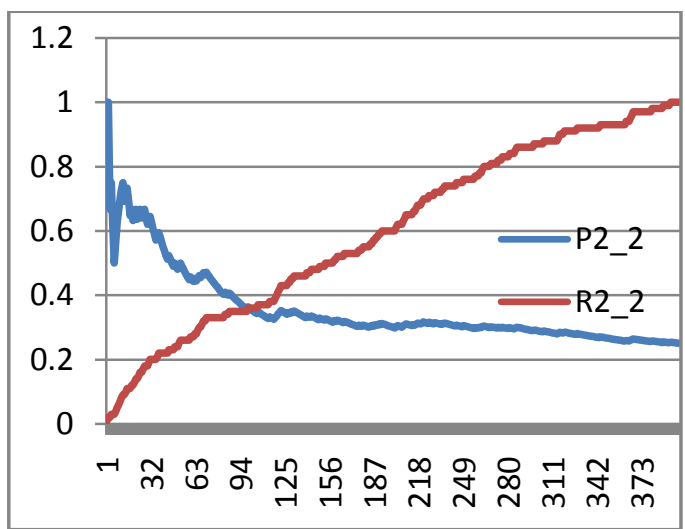

Fig 16: PRCS of class2

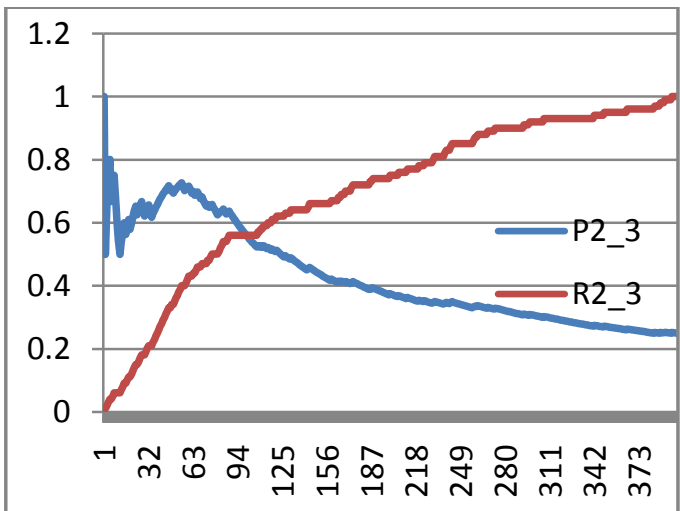

Fig 17: PRCS of class3 


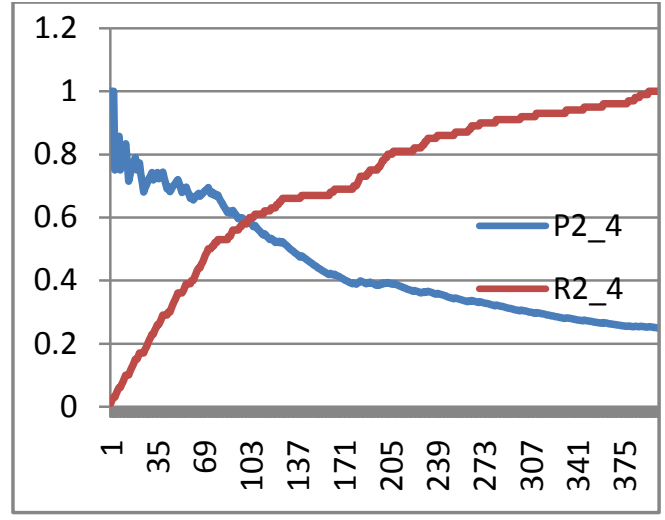

Fig 18: PRCS of class4

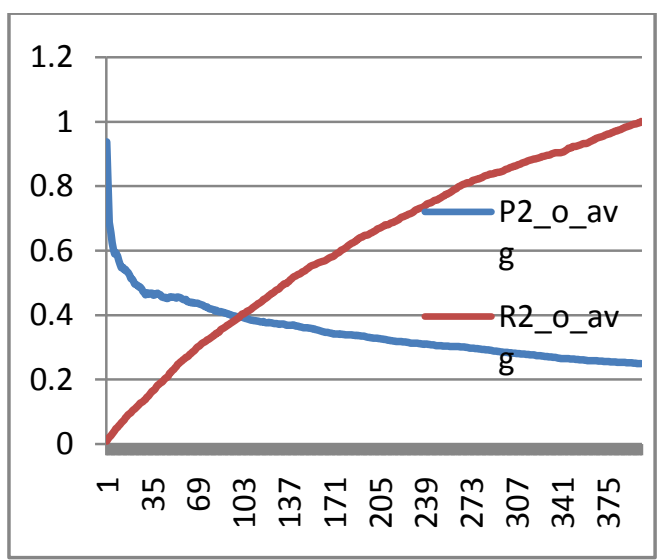

Fig 19: PRCS of Overall Result

\subsubsection{Euclidian Distance Method}

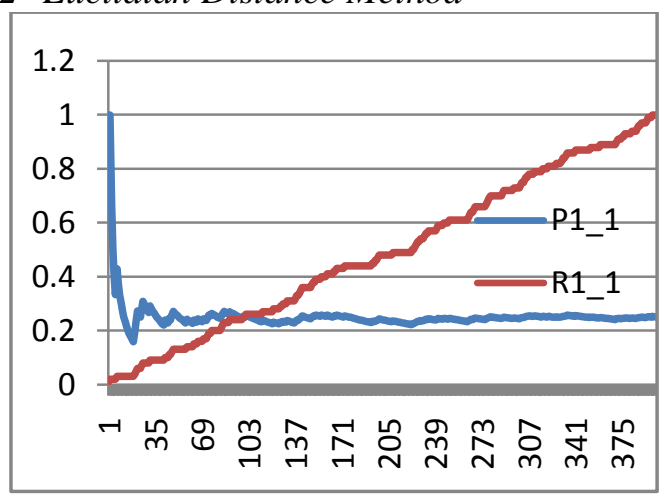

Fig 20: PRCS of class1

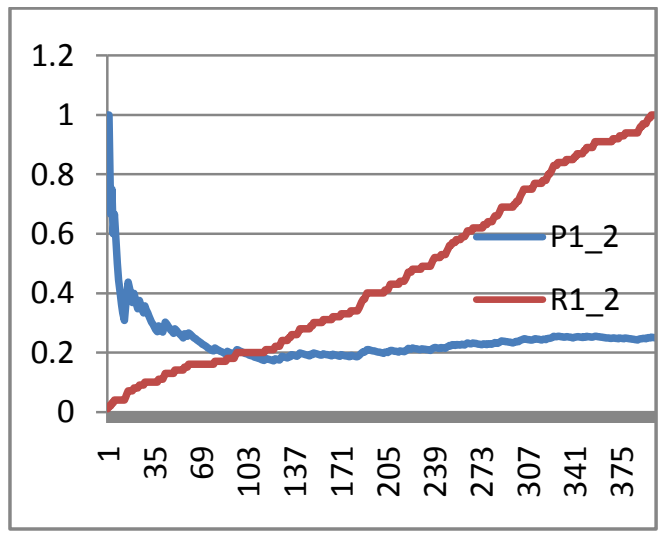

Fig 21: PRCS of class2

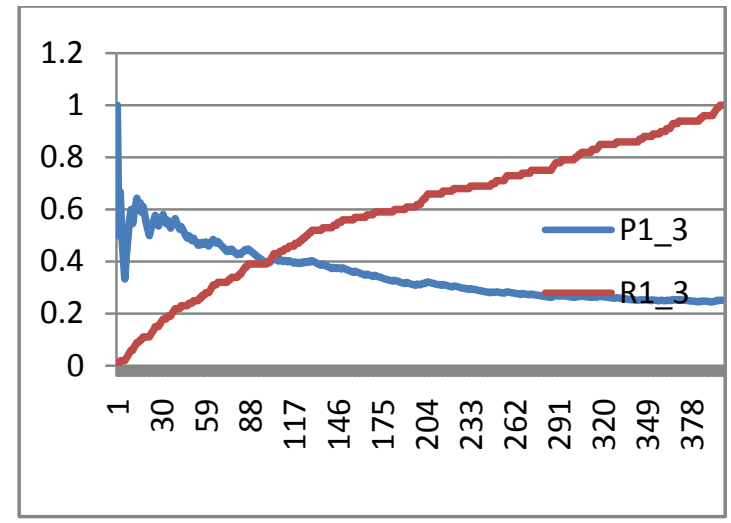

Fig 22: PRCS of class3

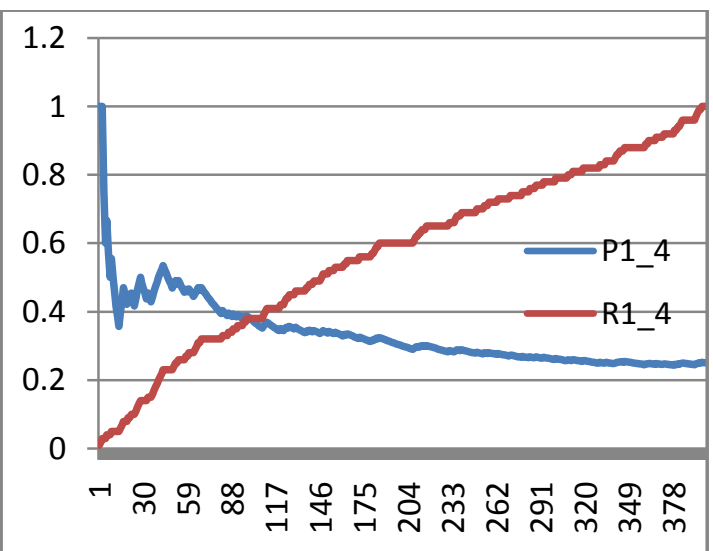

Fig 23: PRCS of class4

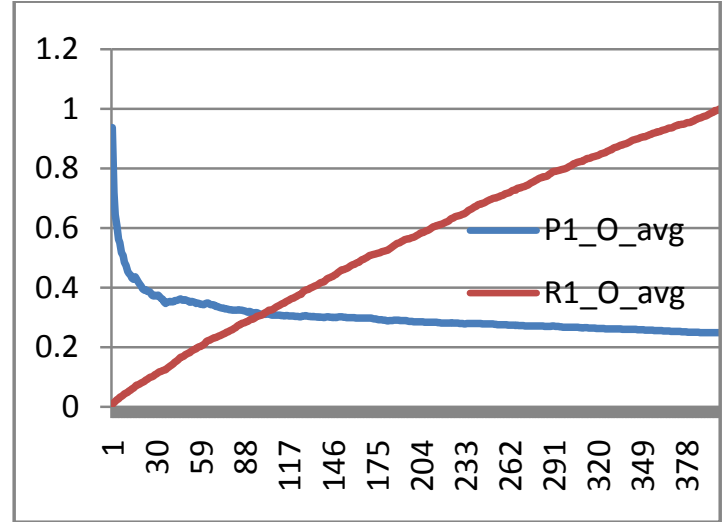

Fig 24: PRCS of Overall Result

By combining DCT and kekre's transform different result sets are available. When this combination is used with absolute distance method then generated result for class 1 was around $40 \%$, class $240 \%$, class $340 \%$ and class 4 around $60 \%$ (Fig 58 ). By generating overall result, this approach gives $40 \%$ result (Fig 9). Same approach gives different result when using Euclidian distance method. It gives result for class 1 $20 \%$, class $22 \%$, class $330 \%$ and class 4 around $40 \%$ result (Fig 10-13). The overall result of this approach is around 35\% (Fig 14). Other approach which is combined together is DST and kekre's transform. This also generates different result with different distance calculation methods. Along with absolute distance it generates following results. For classs 1 $50 \%$, class 2 around $40 \%$, class 3 around $60 \%$ and for class 4 $60 \%$ (Fig 15-18). Overall result generated by this approach is more than 40\% (Fig 19). When Euclidian distance method is applied then it gives result for class 1 is $22 \%$, class $220 \%$, 
class 3 more than $40 \%$ and class 4 40\% (Fig 20-23). Overall result is more than $35 \%$ (Fig 24).

\section{CONCLUSION}

Many algorithms were studied in this paper as a reference. When histogram matching of spatial domain is used, it produces better result but it will generate improper result if background or colour combination of different images match. As researchers already worked on frequency domain but they didn't tried for combining the transform methods to enhance the system performance. So this paper combines the different transforms to generate feature vector table and on this basis many result were generated. By observing above graphs and results, simple analysis says that when absolute distance method is used for feature distance calculation it gives better result than when Euclidian distance is used for feature distance calculation. Fig 4 shows Hybrid approach DCT-DST gives $40 \%$ overall result in Euclidian distance while 30\% overall result is achieved by using absolute distance method. While combining DCT-Kekre's transform method overall $40 \%$ matching result were generated. It gives best performance for class 4 i.e. around $60 \%$ matching result. While applying Euclidian distance method this algorithm results around $35 \%$ overall matching result while it gives best result for class 4 which lies around $40 \%$ matching result. DST-Kekre's transform methods are clubbed together to generate best possible result, it was tested with Euclidian distance and $30 \%$ overall matching result is achieved whereas best result is $40 \%$ matching result in two classes. When absolute distance calculation method was applied on this algorithm then overall result above $40 \%$ matching is achieved. But this gives much better result from other methods; it gives better result in every class and result matches $60 \%$. So one can conclude that using frequency domain generates better result as compared to spatial domain. In spatial domain also combination of transforms gives better result. Based on above comparison one can say that DST-kekre's transform generates better result when it is used with the absolute distance method.

\section{REFERENCES}

[1] H. B. Kekre, Dhirendra Mishra, "DCT Sectorization for Feature Vector Generation in CBIR" International Journal of Computer Applications (0975 - 8887) Volume 9- No.1, November 2010.

[2] H. B. Kekre, Dhirendra Mishra, "DCT-DST Plane sectorizationof Row-wise Transformed color Images in CBIR", International Journal of Engineering Science and Technology Vol. 2 (12), 2010, 7234-7244.

[3] H. B. Kekre, Dhirendra Mishra, ChiragThakkar, "Column wise DCT plane sectorization in CBIR," International Journal of Computer Science and Information Technologies (IJCSIT), vol. 3 no. 1, pp. 3229-3235, 2012.

[4] H.B.Kekre, Kamal Shah, "Application of DCT row and column feature vector for face recognition with comparison to full DCT and PCA", International Journal of Computer Applications in Engineering, Technology and Science (IJ-CA-ETS), Vol. 1, No.2, 435-439 April/September 2009.

[5] Dr. H. B. Kekre, Dhirendra Mishra, "Sectorization of Walsh and Walsh Wavelet in CBIR", International Journal on Computer Science and Engineering (IJCSE) Vol. 3 No. 6 June 2011.

[6] H.B.Kekre, Dhirendra Mishra, "Sectorization of Haar and Kekre's Wavelet for feature extraction of color images in image retrieval", International journal of computer science and information security (IJCSIS), USA, Vol.9, No.2, Feb 2011, pp.180-188.

[7] Rui, Yong, Thomas S. Huang, and Shih-Fu Chang. "Image retrieval: Current techniques, promising directions, and open issues." Journal of visual communication and image representation 10, no. 1 (1999): 39-62.

[8] Mandal, Mrinal K., F. Idris, and Sethuraman Panchanathan. "A critical evaluation of image and video indexing techniques in the compressed domain." Image and Vision Computing 17, no. 7 (1999): 513-529.

[9] [9] Sharma, Neetu S., Paresh S. Rawat, and Jaikaran S. Singh. "Efficient CBIR using color histogram processing." Signal \& Image Processing 2, no. 1 (2011).

[10] Jain, Monika, and S. K. Singh. "A survey on: content based image retrieval systems using clustering techniques for large data sets." International Journal of Managing Information Technology (IJMIT) 3, no. 4 (2011): 23-39.

[11] Yan, Chunlai. "Accurate Image Retrieval Algorithm Based on Color and Texture Feature." Journal of Multimedia 8, no. 3 (2013): 277-283.

[12] Gupta, Vaibhav, and Anil Ramawat. "EVALUATION OF CBIR APPROACHES FOR DIFFERENTLY SIZED IMAGES." International Journal on Computer Science and Engineering 4, no. 1 (2012).

[13] Datta, Ritendra, Dhiraj Joshi, Jia Li, and James Z. Wang. "Image retrieval: Ideas, influences, and trends of the new age." ACM Computing Surveys (CSUR) 40, no. 2 (2008): 5.

[14] Tamura, Hideyuki, Shunji Mori, and Takashi Yamawaki. "Textural features corresponding to visual perception." Systems, Man and Cybernetics, IEEE Transactions on 8, no. 6 (1978): 460-473.

[15] Smeulders, Arnold WM, Marcel Worring, Simone Santini, Amarnath Gupta, and Ramesh Jain. "Contentbased image retrieval at the end of the early years." Pattern Analysis and Machine Intelligence, IEEE Transactions on 22, no. 12 (2000): 1349-1380.

[16] Kekre, Dr HB, Sudeep D. Thepade, and Akshay Maloo. "Query by Image Content Using Colour Averaging Techniques." Engineering journals, International Journal of Engineering, Science and Technology (IJEST) 2, no. 6 (2010): 1612-1622.

[17] Kekre, H. B., and Sudeep D. Thepade. "Rendering Futuristic Image Retrieval System." In National Conference on Enhancements in Computer, Communication and Information Technology, EC2IT2009, pp. 20-21. 2009.

[18] Khokher, Amandeep, and Dr Rajneesh Talwar. "Image Retrieval: A State Of The Art Approach For Cbir." International Journal of Engineering Science And Technology (IJEST) (2011).

[19] Shriram, K. V., P. L. K. Priyadarsini, and V. Subashri. "An Efficient and Generalized approach for Content Based Image Retrieval in MatLab." International Journal of Image, Graphics and Signal Processing (IJIGSP) 4, no. 4 (2012): 42.

[20] Kekre, H. B., Sudeep D. Thepade, and Akshay Maloo. "Performance Comparison of Image Retrieval Using Fractional Coefficients of Transformed Image Using DCT, Walsh, Haar and Kekre's Transform." CSCInternational Journal of Image processing (IJIP) 4, no. 2 (2010): 142-155. 